\title{
Ilegalidad de la tenencia y desigualdad en la distribución de la tierra en Ecuador como condiciones de vulnerabilidad
}

\section{Desigualdade na distribuição e ilegalidade na posse da terra no Equador como determinantes da vulnerabilidade.}

\section{Inequality in Distribution and Illegality in Land Tenure in Ecuador as Vulnerability Determiners.}

\author{
Julio Ramiro León Paz ${ }^{1}$ http://orcid.org/0000-0002-6278-7852 \\ Amanda Rivera ${ }^{2}$ http://orcid.org/0000-0002-9162-7698
}

\footnotetext{
${ }^{1}$ PósGrado em Geografia - Pontificia Universidad Católica del Ecuador - Quito, Equador y Universidad Andina Simón Bolívar Quito, Equador email: ramiroleonpaz@gmail.com

${ }^{2}$ Ingeniera ambiental-Universidad Andina Simón Bolívar-Quito, Equador- email . escenario77@gmail.com
}

\section{Resumen}

El artículo aborda la inequidad en la repartición y la ilegalidad en la tenencia de la tierra en el Ecuador y como estos factores son condicionantes de vulnerabilidad, tomando en cuenta dos casos de estudio específicos como son los cantones de Pedernales en la provincia de Manabí y de Rioverde en la provincia de Esmeraldas. Se utilizó una metodología de trabajo empírica con levantamiento de campo insitu e investigativa con la aplicación del Modelo PAR (Presión y Liberación de los Desastres) y el coeficiente de GINI, para la tenencia de la tierra, también se observa que las presiones dinámicas del territorio afectan las población económicamente menos favorecida, concluye que la gestión adecuada de la tierra es una acción que permitirá minimizar el riesgo existente y reducir el riesgo a futuro.

Palabras clave: Inequidad. Vulnerabilidad. Reducción de Riesgos.

\section{Resumo}

Este artigo trata da desigualdade na distribuição e ilegalidade da posse da terra no Equador e como esses fatores são fatores determinantes da vulnerabilidade, levando em consideração dois estudos de caso específicos, como os cantões de Pedernales, na província. Manabí e Rioverde, na província de Esmeraldas. Utilizou-se uma metodologia empírica de trabalho com uma pesquisa de campo in situ e investigativa, com a aplicação do Modelo PAR (Pressão e Liberação Desastres) e o coeficiente GINI, para a posse da terra, também é observado que as pressões dinâmicas do território afetam a população economicamente menos favorecida em uma porcentagem maior, conclui-se que o manejo adequado da terra é uma ação o que minimiza o risco existente e reduz o risco futuro.

Palavras-chave: Desigualdade. Vulnerabilidade. Redução de risco.

\footnotetext{
Abstract

This article deals with inequality in the distribution and illegality of land tenure in Ecuador and how these factors are determinants of vulnerability, taking into account two specific case studies, such as the cantons of Pedernales, in the province. Manabí and Rioverde, in the province of Esmeraldas. An empirical methodology of work was used with an in situ and investigative field research, with the application of the PAR Model (Pressure and Disaster Release) and the GINI coefficient, for land tenure, it is also observed that the dynamic pressures of the territory affect the economically less favored population in a higher percentage, it is concluded that the proper management of the land is an action that minimizes the existing risk and reduces the future risk.
} 
Keywords: Inequity. Vulnerability. Reduction of Riesgos.

\section{Introducción}

La inequidad en el proceso de repartición de la Tierra en el Ecuador se ha venido dando a lo largo de la historia, principalmente con el aparecimiento de los booms económicos relacionados a la agro exportación y a la extracción del petróleo, lo que dio como resultado el apoderamiento de grandes extensiones de terreno por parte de las familias más acaudaladas del país, ocasionando el asentamiento irregular alrededor de las haciendas de pobladores que trabajaban dentro de las mismas; el principal objetivo de este artículo es dar a conocer como la inequidad en la repartición de la tierra y la ilegalidad de posesión de la misma, son factores subyacentes que se convierten en condicionantes de vulnerabilidad para la población aumentando el riesgo de desastres en el país.

El artículo planteado se basa en el análisis de la inequidad en la repartición de la tierra y la ilegalidad en la tenencia de la misma como condicionantes de vulnerabilidad, por lo que se analizara el porcentaje de legalidad de las tierras en los cantones de Rioverde y Pedernales y el por qué hay niveles tan bajos de legalización, además de observar cómo afecta al desarrollo social la inequitativa repartición de recurso tierra, dichos resultados servirán para poder evidenciar la problemática y motivar a que los gobiernos cuenten con información sobre la propiedad de la tierra, que ayuden a aumentar el porcentaje de legalidad en la tenencia de la tierra, una equitativa repartición de los recursos y establecer medidas de reducción de riesgos, respuesta y recuperación post desastre por lo que se ha hecho un trabajo de levantamiento de datos en campo e investigativo en gabinete para así poder establecer la correlación de estas dos variables en el aumento de la vulnerabilidad. 
El Ecuador es uno de los países más pequeños de América del Sur, sin embargo tiene uno de los índices más altos de inequidad en el acceso de la tierra con un coeficiente de Gini de 0.81 , de acuerdo a lo descrito anteriormente se observa que la ilegalidad en la tenencia de la tierra es un problema más crítico aún ya que en poblaciones que fueron afectadas por el Terremoto del 16 de abril del 2016, como el caso de Pedernales en la provincia de Manabí tan solo el 35\% de los predios afectados tenían escritura, el mismo caso se da en el cantón Rioverde en la provincia de Esmeraldas donde tan solo el 23\% de los predios están legalizados.

Ahora bien, si se entiende que la vulnerabilidad es un proceso eminentemente social por lo que hace referencia a las características de una población o sistema, que los hace susceptibles a los efectos dañinos de una amenaza y lo relacionamos con lo descrito en párrafos anteriores podremos visualizar que la falta de acceso al recurso, en este caso la tierra y la necesidad de recibir servicios como educación, salud o conectividad han hecho que la población menos favorecida se ubique en lugares con un mayor grado de exposición ante las amenazas naturales preexistentes mediante invasiones y asentamientos ilegales.

Ecuador en la última década se caracterizó por la construcción de grandes obras de infraestructura en ejes como salud y educación, teniendo la particularidad que se ubicaban en los centros poblados principales, por lo que se eliminaron los servicios de este tipo en las comunidades vecinales casi obligando de manera involuntaria a un traslado de la población buscando estos beneficios para sus familias generando condiciones de vulnerabilidad social ante las amenazas naturales existentes.

La inequidad e ilegalidad de la tierra en nuestro país es un condicionante de vulnerabilidad debido a que expone a los elementos ante posibles eventos peligrosos que de un simple proceso natural termina siendo un desastre. Una mejor distribución del recurso tierra es una acción para reducir el riesgo presente y minimizar el riesgo futuro.

\section{Inequidad: marco teórico}

La Inequidad se defina como la diferencia existente entre los grupos sociales que conforman una sociedad, la desigualdad de oportunidades para poder acceder a recursos y servicios como vivienda, educación o salud y como resultado de esto la ausencia de un desarrollo para poder tener una vida digna.

Los polos de desarrollo son zonas geográficas relativamente reducidas en las que se estimula la localización de actividades industriales para que impulsen la actividad 
económica en un área geográfica de mayor amplitud. Aunque con algunos matices diferenciadores, también se denominan polos de crecimiento y polos de promoción industrial, ahora bien tomando en cuenta la evolución urbana del Ecuador se localiza dos Polos de desarrollo históricamente bien definidos tanto en la sierra como en la costa, que son Quito y Guayaquil, los mismos que han sido ejes en el desarrollo del resto de ciudades cercanas a ellas.

La Unidad de Producción Agropecuaria -UPA se refiere a una extensión de tierra de $500 \mathrm{~m}^{2}$ o más, dedicada total o parcialmente a la producción agropecuaria, considerada como una unidad económica.

Según el EIRD ${ }^{\mathrm{i}}$ - 2009 la vulnerabilidad se define como las características y circunstancias de una comunidad, sistema o bien, que los hacen susceptibles a los efectos dañinos de una amenaza, hay que tomar en cuenta que tanto la exposición como la vulnerabilidad son resultados de determinadas acciones humanas o procesos sociales. El UNISDR ${ }^{\text {ii }-2009 ~ d e f i n e ~ l o s ~ d e s a s t r e s ~ c o m o ~ u n a ~ g r a v e ~ i n t e r r u p c i o ́ n ~ e n ~ e l ~}$ funcionamiento de una comunidad o sociedad que ocasiona una situación generalizada de impactos y pérdidas humanas, materiales, económicas y ambientales; que exceden la capacidad de la comunidad o la sociedad afectada para hacer frente a la situación mediante el uso de sus propios recursos.

Se entiende por amenaza como un fenómeno, sustancia, actividad humana o condición peligrosa que puedan ocasionar la muerte, lesiones u otros impactos a la salud, al igual que daños a la propiedad, la perdida de medios de sustento y de servicios, trastornos sociales y económicos o daños ambientales (EIRD- 2009).

El riesgo de desastre se define como las perdidas probables que ocasionaría un desastre, en términos de vidas humanas, las condiciones de salud, los medios y los servicios, que pueden ocurrir en una comunidad o sociedad en particular en un periodo especifico de tiempo en el futuro (EIRD- 2009).

La gestión de riesgo de desastre definida en forma genérica se refiere a un proceso social cuyo fin último es la prevención, la reducción y el control permanente de los factores de riesgo de desastre en la sociedad, en consonancia con, e integrada al logro de pautas de desarrollo humano, económico, ambiental y territorial, sostenibles.

El coeficiente de gini es un indicador utilizado para medir el nivel de desigualdad en el acceso al recurso existente entre los habitantes de una región, es decir el coeficiente demuestra hasta qué punto la distribución de ingreso entre individuos u hogares o el acceso a los principales recursos dentro de una economía se aleja de la 
distribución equitativa, cuando el resultado se acerca más a 1 quiere decir que existe una mayor desigualdad en la repartición de los recursos.

El modelo par, modelo de presión y liberación de los desastres, explica la forma como los factores subyacentes y causas de fondo incorporadas en la vida diaria dan origen a presiones dinámicas que afectan grupos particulares y conducen a condiciones específicamente inseguras; tomando en cuenta que dos dinámicas generan el riesgo de desastre y en su caso extremo el desastre, la amenaza y la vulnerabilidad creciente.

\section{La tenencia de la tierra en el Ecuador a lo largo de la historia.}

En nuestro país los procesos de apoderamiento de la tierra inicia en la época de la colonización española, a medida que pasaron los años el sistema de apropiación arbitraria de las tierras se intensificó principalmente en la sierra cuando se obligó a los indígenas a retirarse de los valles llevándolos a ubicar en las zonas de montaña, el resultado de la conformación y ubicación en el territorio al final del período colonial, muestra una red de ciudades establecidas en la sierra, demostrando una tendencia hacia las desigualdades en la tenencia de la tierra tanto en sus dimensiones como en la calidad de la misma.

Para inicios del siglo XX el avance del modelo agroexportador y la intensificación de la producción de banano, café y cacao en la costa ecuatoriana genera que los grupos económicos más fuertes acaparen el recurso tierra dejando sin acceso a los menos favorecidos, además existe un cambio significativo en la relación de la población con el territorio, debido a que estos procesos de crecimiento económico desenfrenado, dan como resultado la formación de nuevos asentamientos cercanos a las zonas de producción convirtiendo así el paisaje en ciudades flotantes que dependen de la cosecha, y que con el paso del tiempo se convierten en ciudades establecidas pero sin servicios y principalmente asentados en zonas de riesgo.

La tenencia de la tierra en el país siempre ha estado ligada a los grandes booms económicos que han dado a lo largo de la historia, a mediados de siglo pasado con la extracción del petróleo se dio un poblamiento en la zona limítrofe del país y un cambio en el uso de suelo en la zona causando un deterioro ambiental ya que se transformó grandes cantidades de selva amazónica en pastizales para ganadería.

\section{La Inequidad del acceso al recurso tierra en Ecuador}

En el Ecuador un $98.3 \%$ del recurso tierra se encuentra en manos privadas pero con una desigualdad total de repartición, según datos del Censo Agropecuario del año 
2000, las UPA ${ }^{\text {iii }}$ menores a 5 hectáreas representan al 63,96\% de las UPAS totales pero acceden solamente al 6,53\% de la superficie agrícola del Ecuador, lo que quiere decir que cada una tiene un promedio de 1,4 ha, mientras que las propiedades entre 50 y 100 ha representan el 3,97 ha de las UPA y un $18.33 \%$ de la superficie agrícola, mientras que las propiedades superiores a 500 ha constituyen menos del 1\% de las UPA pero controlan alrededor del $16 \%$ de la superficie agrícola, promediando un tamaño de 1400 ha, lo que hace una diferencia directa de 1000 a 1 entre los propietarios de grandes y pequeñas extensiones de terreno.

La inequidad en la tenencia de tierra en el Ecuador es una de las más altas a nivel de América Latina tomando en cuenta que somos un país relativamente pequeño en comparación con otros de la región, el coeficiente de Gini utilizado en este caso para medir la desigualdad en el acceso al recurso tierra señala um 0.81 lo que es un resultado alarmante, el 99.9\% de las UPA tienen extensiones inferiores a 640 ha, es decir tan solo el $0.01 \%$ tiene extensiones superiores a la mencionada anteriormente.

Mapa 1- Índice de Gini a Nivel Provincial - Equador

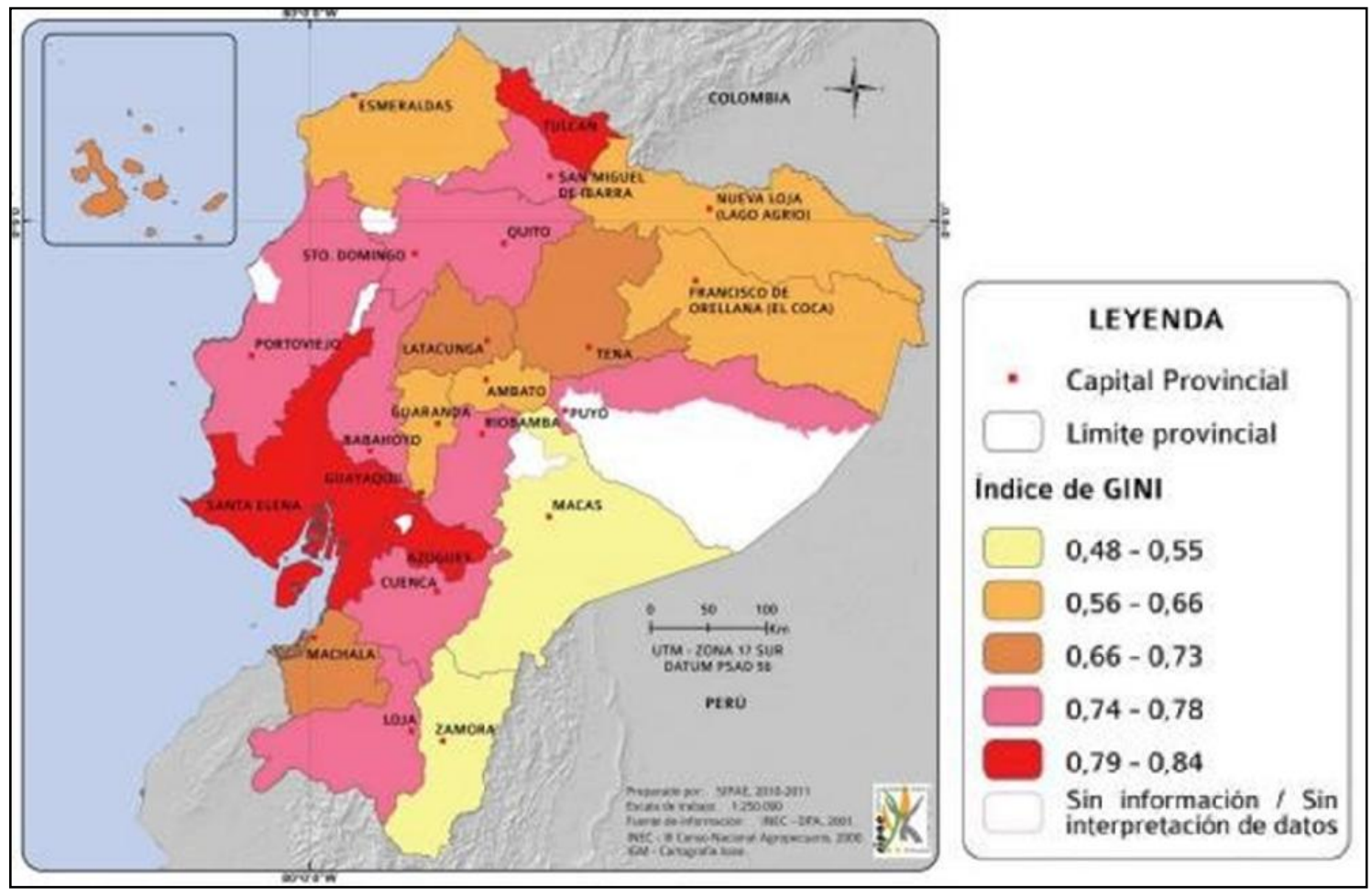

Fuente: Atlas de la Tenencia de la Tierra en Ecuador Elaborado por: SIPAE 2010-2011

La inequidad en nuestro país es tan marcada que la mitad de las UPA tiene una extensión que no permite el desarrollo adecuado de los campesinos, el aumento en la concentración de la tierra en condiciones de presión a los propietarios de pequeñas extensiones de terreno está generando que la población se quede sin tierra literalmente, 
los datos existentes en el III Censo Agropecuario establecen que existen 165.000 UPA que no llegan a 0.5 ha.

Esta situación de inequidad se presenta en el país a pesar de que en este existieron dos Reformas Agrarias entre los años 60 y 70, las mismas tenían el objetivo de frenar el sistema latifundista que había despojado a los indígenas de sus tierras, el principal problema fue que los territorios entregados eran de mala calidad para la agricultura o estaban ubicados en zonas de riesgo, hay que tomar en cuenta que los propietarios de minifundios en muchos casos no tenían acceso al agua de riego por lo que existía una baja productividad por parcela, además de la falta de recursos para la inversión de tecnología para poder mejorar el rendimiento de sus tierras.

\section{La Inequidad en la repartición de le tierra como condicionante de vulnerabilidad.}

Tomando en cuenta que la vulnerabilidad es un proceso eminentemente social, y que un país como el nuestro está rodeado de amenazas naturales de todo tipo, el que un evento peligroso se considere o no riesgo, dependerá directamente de que el territorio donde este ocurra se encuentre poblado, es decir del grado de exposición existente, por lo que se puede decir que los desastres constituyen fenómenos sociales mas no de tipo natural y que evidencian problemas no resueltos del desarrollo.

En muchas poblaciones del país la falta de acceso a los recursos básicos generan condiciones de pobreza extrema, consecuencia de esto presentan una gran dificultad para recuperarse ante un desastre, el fortalecimiento social de estas poblaciones se puede tomar en cuenta como una medida eficaz de reducción del riesgo; pero ante todo lo expuesto la pregunta es ¿Por qué la inequidad en la repartición de la tierra condiciona la vulnerabilidad?, al entender a la vulnerabilidad como un proceso de construcción social nos damos cuenta que toda acción realizada por el ser humano puede aumentar o reducir el riesgo existente.

Como se ha establecido en párrafos anteriores la concentración del recurso tierra en pocas manos ha ocasionado que las poblaciones menos favorecidas se encuentran expuestas a distintas amenazas, como pendientes escarpadas, riveras de ríos, cerca de zona de licuefacción y para lograr estos asentamientos se realiza en muchos casos deforestaciones e impactos negativos al medio ambiente aumentando así el factor de exposición ante las amenazas presentes; la Política de Estado en tema de servicios como educación y salud en los últimos 10 años fue la de mejorar la atención y el sistema en 
general, con la construcción de mega obras centralizadas, principalmente en la temática de educación, se eliminaron las escuelas unidocentes, y se crearon las llamadas "Escuelas del Milenio" en los poblados con mayor población obligando a una migración interna mal llamada voluntaria, puesto que los campesinos para que sus hijos puedan acceder a este recurso debieron movilizarse hacia las centralidades, lastimosamente la tierra ya estaba ocupada, entonces esta población flotante al no tener acceso al recurso se asientan literalmente donde hay espacio, con construcciones precarias y poco técnicas pero con el único objetivo de tener este servicio.

Imagen 1- Modelo de Presión y Liberación del Desastre (PAR)

\begin{tabular}{|c|c|c|c|c|}
\hline $\begin{array}{l}\text { CAUSAS DE } \\
\text { FONDO }\end{array}$ & $\begin{array}{l}\text { PRESIONES } \\
\text { DINAMICAS }\end{array}$ & $\begin{array}{l}\text { CONDICIONES } \\
\text { INSEGURAS }\end{array}$ & DESASTRE & AMENAZAS \\
\hline $\begin{array}{l}\text { Acceso } \\
\text { Limitado a: } \\
\text { Recursos } \\
\text { Tierra, } \\
\text { Sistemas } \\
\text { económicos } \\
\text { de desarrollo }\end{array}$ & $\begin{array}{l}\text { Crecimiento } \\
\text { Poblacional } \\
\text { Urbanismo } \\
\text { Acelerado Falta } \\
\text { de Servicios }\end{array}$ & $\begin{array}{l}\text { AmbienteFrágil: } \\
\text { Localización peligrosa. } \\
\text { Infraestructura poco } \\
\text { técnica. } \\
\text { Economía Frágil: } \\
\text { Bajos ingresos lo que } \\
\text { conlleva una precariedad en } \\
\text { la vida } \\
\text { Sociedad Vulnerable: } \\
\text { Grupos vulnerables en } \\
\text { riesgo. } \\
\text { Acciones Públicas: } \\
\text { Falta de personal } \\
\text { capacitado y acciones de } \\
\text { prevención }\end{array}$ & $\begin{array}{l} \\
\text { RIESGO } \\
\text { AMENAZA } \\
\mathbf{X} \\
\text { VULENRABIL } \\
\text { IDAD } \\
\end{array}$ & $\begin{array}{c}\text { TERREMOTOS } \\
\text { INUNDACIONE } \\
\text { S ERUPCIONES } \\
\text { VOLCANICAS } \\
\text { DESLIZAMIEN } \\
\text { TOS SEQUIAS }\end{array}$ \\
\hline
\end{tabular}

Fuente: El Autor (2019)

El mismo caso se presenta en la ganadería y agricultura, como las "buenas" tierras ya están ocupadas se realiza sembríos poco técnicos en pendientes lo que ocasiona que el suelo pierda su compactación y se erosione con las pisadas del ganado lo que conlleva a aumentar el riesgo de deslizamientos de tierra y crezca la vulnerabilidad de las poblaciones asentadas en las faldas de las montañas, otro problemática que se ha presentado en la última década es el crecimiento población y la expansión urbana desenfrenada, la falta de productividad por la mala calidad de las tierras hace que la gente migre y los terratenientes han cambiado el uso de suelo a un sistema de urbanización, dividiendo y vendiendo lotes pequeños en su mayoría que no exceden los 300mts, sin la entrega de servicios básicos como dicta la ley, pero la falta de organismos de control hace que no exista obligatoriedad en estos procesos.

La mayoría de estos predios se encuentran en zonas inseguras, pero por la necesidad, desesperación y falta de información de las personas con menos recursos los 
adquieren, en muchos casos, si no es en todos, se convierten en asentamientos irregulares debido a su imposibilidad de ser legalizados por su ubicación de esta manera existe un crecimiento de la vulnerabilidad física por exposición territorial y la vulnerabilidad social por todos los fenómenos que conllevan este tipos de asentamientos.

\section{La ilegalidad en la Tenencia de la Tierra como condicionante de vulnerabilidad}

La ilegalidad en la tenencia de la tierra es uno de los principales problemas de nuestro país en temas catastrales, desde las herencias sin sustento hasta la venta de terrenos por los traficantes de tierra, son obstáculos con los que tienen que lidiar día a día los distintos GADM's del Ecuador, las invasiones a causa de la falta de acceso al recurso y luego la exigencia de los usuarios para que dichas tierras sean legalizadas y se entreguen todos los servicios básicos causan un freno en el desarrollo territorial.

\section{Caso Pedernales}

La ilegalidad en la tenencia no era tomada en cuenta al momento de hablar de Gestión de Riesgos en nuestro país, a partir del 16 de abril de 2016 cuando ocurrió el terremoto en las provincias de Esmeraldas y Manabí, mismo que causo una seria afectación a toda la zona costera del Ecuador.

Tabla 1- Población y Número de predios por parroquia

\begin{tabular}{c|c|c|r}
\hline $\begin{array}{c}\text { CABECERA CANTONAL Y } \\
\text { PARROQUIALES }\end{array}$ & $\begin{array}{c}\text { NUMERO DE } \\
\text { HABITANTES }\end{array}$ & $\begin{array}{c}\text { SUPERFICIE } \\
\text { POR (has) }\end{array}$ & $\begin{array}{c}\text { NUMERO DE } \\
\text { PREDIOS }\end{array}$ \\
\hline Pedernales & 24073 & 1510 & 9705 \\
\hline Cojimíes & 6165 & 173 & 4134 \\
\hline 10 de Agosto & 210 & 26.36 & 144 \\
\hline Atahualpa & 232 & 16.84 & 163 \\
\hline TOTAL & 30680 & 1726.20 & $\mathbf{1 4 4 6 4}$ \\
\hline
\end{tabular}

Fuente: PROYECCIÓN INEC 2015. CENSO 2010. IEE, SENPLADES, IGM

Las autoridades se dieron cuenta del serio obstáculo que presentaba la ilegalidad de la tenencia de la tierra, según datos entregados por el MIDUVI ${ }^{\text {iv }}$ unos meses después del desastre se pudo determinar que el $53 \%$ de los predios afectados no estaban legalizados o catastrados, por lo que la entrega de ayuda para nuevas viviendas o para reparación de afectaciones leves en las construcciones se tuvieron que retrasar hasta solucionar el primer problema, aumentando la vulnerabilidad de la población que ya se encontraba en hogares temporales luego del desastre. 
PAZ,R.L. RIVERA, A.

El aumento poblacional del cantón es producto de la acción de los traficantes de tierras, cuyo modo de operación era el de promocionar a Pedernales cómo un lugar de grandes oportunidades laborales con la finalidad de ofrecer terrenos a precios asequibles. El recurso suelo invadido pertenecía a personas que no residían permanentemente en Pedernales, situación que facilitaba su ilegal posesión, a diferencia de varias familias terratenientes que poseen grandes extensiones de tierra. Los asentamientos humanos irregulares, la mayoría ubicados en zonas de riesgos, la construcción precaria obviando las normativas y procesos adecuados de construcción han llevado a que durante años se vaya gestando un contexto altamente vulnerable, que por falta de previsión, decisión política ocasionó que las invasiones se apropiaran del territorio.

Mapa 2 - Viviendas semaforizadas en la zona urbana del Cantón Pedernales

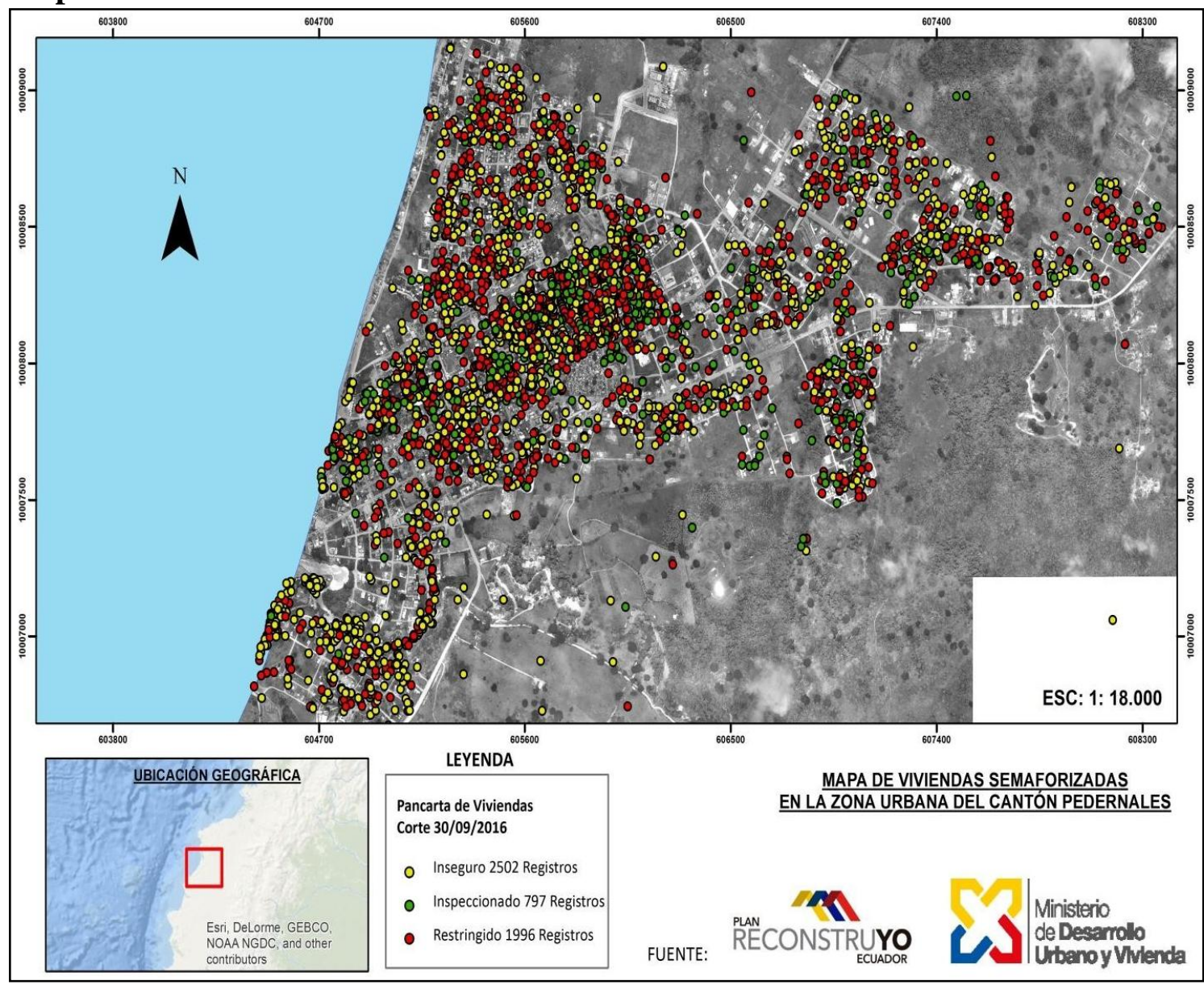

Fuente: Cartografía Básica del INEC, IGM, SNI, MIDUVI, SENPLADES 2016 Elaborado por: Unidad de Diseño \#1 Pedernales

Como se observa en el mapa número 2, la afectación con daños considerables, catalogados como inseguros o restringidos suman un numero de 4998 registros que representan al 50\% del total de predios de la zona urbana, de estos tan solo el $47 \%$ 
tenían posesión legal y registrada en el Departamento de Avalúos y Catastros del Municipio de Pedernales, por lo que se determina que más 3000 predios afectados sin registro, como se mencionó en párrafos anteriores, el problema salió a la luz luego del sismo, instituciones nacionales e internacionales se encontraron con un serio problema al momento de entregar la asistencia a los damnificados

Las primeras acciones que se tomaron fue el de legalizar todos estos predios olos que más se pudo como manifestó la persona encargada del avalúos del GAD, a otros tantos que no se pudo legalizar porque estaban ubicados en invasiones se les entrego predios y casa básicamente en el sector "La Chorrera" que después de tres años del desastre aún no se ha podido legalizar del todo dicha situación.

Otra problemática como ya se hablo es la falta de accesibilidad al recurso, en la cabecera cantonal de Pedernales se construyeron dos escuelas del milenio, lo que obligo a los pobladores de comunidades como: La Playita, San Vicente, Pata de Pájaro, Tachina, entre otros tuvieron que desplazarse hacia la centralidad o cerca de la misma formando asentamientos irregulares para poder acceder al servicio, por la precariedad de las construcciones el momento del sismo todas fueron afectadas, dejando a estas personas a la intemperie, en busca de ayuda fueron ubicadas en los alojamientos temporales lo que al final ocasiona un perjuicio para el Estado y para la población en sí.

\section{Caso Rioverde}

El crecimiento poblacional de las ciudades costeras en el Ecuador se ha dado de manera desorganizada, siempre caracterizada por asentamientos irregulares y anclados a los distintos booms económicos que han aparecido a lo largo de la historia, se han identificado con los crecimientos económicos de los grandes terratenientes, generando asentamientos en las cercanías de haciendas o pequeños poblados de pescadores.

Dicha ubicación geográfica de estos poblados han dado como resultado pequeñas ciudades con carencias de servicios básicos y una alta inseguridad ante las amenazas naturales propias de los procesos geomorfológicos que se dan en el litoral.

Según el Plan de Desarrollo y Ordenamiento Territorial la zona urbana cuenta con una extensión de 123,63 has, y una proyección de expansión de la misma dando una totalidad de 317,53 has, hay que recalcar que las cabeceras parroquiales fueron declaradas zonas urbanas, pero no cuentan con la información cartográfica necesaria. 
Mapa 3- Multiamenazas del Cantón Rioverde

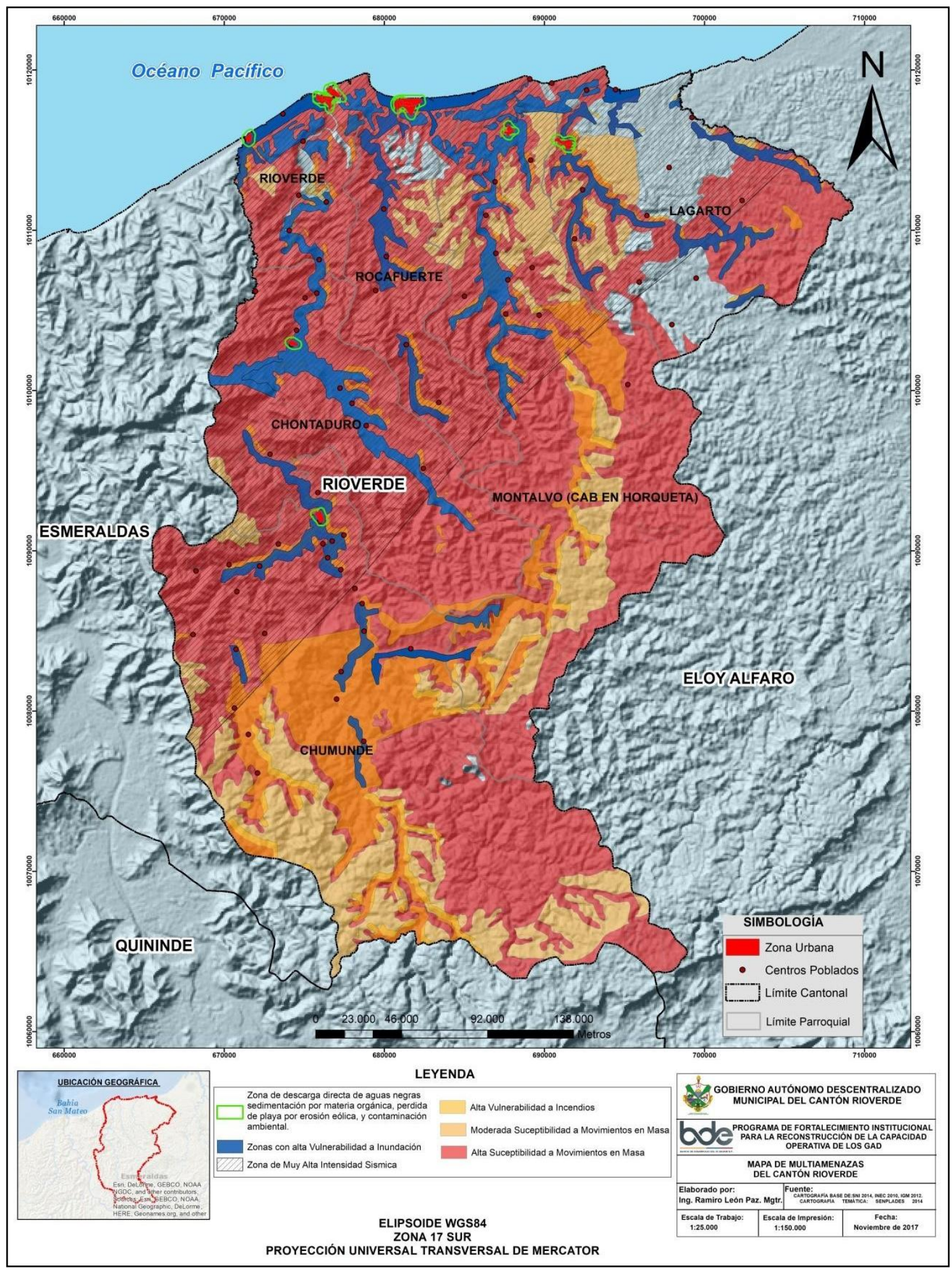

Fuente: Cartografía Básica del INEC,BDE, IGM, SNI, MIDUVI, SENPLADES 2016 Elaboración propia.

Al no poseer la cartografía para trabajar en el catastro rural, se ha optado por ingresar en un inicio solo los predios que son escriturados por sus propietarios sin 
existir aun la obligatoriedad de catastrarlos, en cantón se encuentran legalizados apenas el $23 \%$ del total de sus predios tanto en la zona urbana como en la zona rural lo que refleja un alto porcentaje de ilegalidad en la tenencia y la presencia de un sinnúmero de asentamientos considerados irregulares.

En este cantón la tenencia de la tierra en pocas manos dedicadas en su gran mayoría a la ganadería y la industria camaronera ha dado como resultado el crecimiento de asentamientos irregulares cerca de las grandes haciendas principalmente a lado de las vías principales, en la parroquia de Montalvo, comunidades como Walte, La Muralla, Machin, ubicadas en las zonas altas del cantón, por falta de trabajo su población se ha terminado desplazando para buscar trabajos temporales, asentándose cerca de las riveras del Río Verde, aumentando la vulnerabilidad en caso de la amenaza latente de inundaciones.

El caso de la parroquia Chontaduro es diferente ya que por estar dedicada en su gran mayoría a la agricultura de subsistencia la migración se ha dado por otras circunstancias, cuando el Gobierno Nacional decidió cerrar las escuelas unidocentes, comunidades como Las Cumbres, Chorreron, Papayal quedaron aisladas de recibir este servicio teniendo que caminar hasta 4 horas al día para poder llegar a clases, por lo que muchos pobladores realizaron invasiones cercanas a los caminos de acceso, que no pudieron ser controladas por el GAD, y que a lo largo del tiempo se ha transformado en un serio problema, tanto en el ámbito privado como en el público.

Dichos asentamientos por ser irregulares se han asentado en las riberas de los ríos y en pendientes pronunciadas donde son altamente vulnerables por exposición física, especialmente en la temporada invernal que en este cantón suele ser muy severa, según datos entregados por la jefatura de riesgos del GAD Municipal solo este año se ha perdido cerca de 10 vidas y cuantiosas cifras económicas por los deslizamientos de tierra en asentamientos irregulares.

\section{Consideraciones finales}

Según la Constitución de la República del Ecuador del año 2008 la Gestión de Riesgo debe ser tomada como una transversalidad en la ejecución de proyectos y principalmente en el Ordenamiento Territorial de los GAD, por lo que es de suma importancia comenzar a trabajar de manera exhaustiva en una ley que regule la gestión de riesgos en el áís o legislación asociada, que contemple temas de tenencia de tierras en 
situaciones de desastres y facilite la aplicación de directrices dentro del GAD para que fortalezcan esta temática.

La ilegalidad en la tenencia de la tierra es uno de los problemas más graves que tiene el Ecuador, más aun cuando este condiciona la vulnerabilidad creciente ante las amenazas existentes, post desastre los procesos de reconstrucción o entrega de ayuda para reconstrucción se vuelven casi imposibles por la irregularidad de los asentamientos.

Los GAD’s deben hacer campañas masivas de legalización de tierras que sirvan para ayudar a los menos favorecidos y de esta manera mejorar el desarrollo del territorio en el que se encuentran.

La Inequidad en la repartición de la riqueza y de los recursos naturales han condenado a países enteros a la pobreza generalizada, y más aún en el tema de la tierra a poblaciones menos favorecidas a exponerse ante los fenómenos naturales en búsqueda de un mejor estilo de vida, se debe establecer lineamientos a nivel nacional sobre la tenencia y ocupación de la tierra pública y privada.

Se debe generar Planes de Gestión de Riesgos para poder minimizar el riesgo existente en localidades asentadas en zonas de riesgo y darles soluciones para que puedan llevar un modo de vida digno.

Para todos los asentamientos que se deben regularizar o desplazar se tienen que tomar en cuenta los medios y los modos de vida de estas personas para así no transgredir sus derechos fundamentales.

Los proyectos de desarrollo urbano deben ir de la mano con la temática de gestión de riesgos para que tenga un funcionamiento adecuado y cuando existan eventos peligrosos no ocurra una pérdida de infraestructura o vidas, y que los recursos invertidos en estos proyectos no sea dinero perdido.

\section{Referências}

Abhas K. Jha, Todd W. Miner, and Zuzana Stanton-Geddes (2013). Building Urban Resilience, The World Bank, Sidney

Anne-Catherine Chardon \& Juan Leonardo González (2002). Indicadores para la Gestión de Riesgos, Universidad Nacional de Colombia. Manizales,

Ben Wisner, Piers Blaikie, (2005). At Risk. Routledge, Londres, 
Terry Cannon; Piers Blaikie; Ian Davis (2003). At Risk: Natural Hazards, People's

Vulnerability and Disasters, Routledge, Londres,

BRASSEL, HERRERA y LAFORGE (2008). ¿Reforma Agraria en el Ecuador?: viejos temas, nuevos argumentos. SIPAE. Quito,

Pierre Gondard y Hubert Mazurek, 30 Años de Reforma Agraria y Colonización en el Ecuador (1964-1994): dinámicas espaciales, Estudios de Geografía, Vol. 10, CEN, CGE, IRD, PUCE 2001, p. 15-40.

SIPAE (2011). Atlas, Tenencia de la Tierra en Ecuador, SIPAE. Quito, 